\title{
Ovarian cancer risk and use of phenolphthalein-containing laxatives ${ }^{\dagger}$
}

\author{
Glinda S. Cooper* ${ }^{1}$, Matthew P. Longnecker ${ }^{1}$ and Ruth K. Peters ${ }^{2}$ \\ ${ }^{1}$ Epidemiology Branch, National Institute of Environmental Health Sciences, Durham, NC, USA \\ ${ }^{2}$ Department of Preventive Medicine, USC Keck School of Medicine, Los Angeles, CA, USA
}

\begin{abstract}
SUMMARY
Purpose Experimental studies in rodents demonstrated the carcinogenic potential of phenolphthalein, the active ingredient in some laxatives, administered at doses similar to the dose that could be used by humans. Ovarian cancer was one of the cancers observed in these studies. We examined the association between epithelial ovarian cancer and use of phenolphthalein-containing laxatives in a population-based case-control study.

Methods The study includes 356 epithelial ovarian cancer cases (256 invasive, 100 borderline) and 424 controls. Cases were identified through a population-based registry in Los Angeles County in 1992-1998, and controls were matched to cases by age, race/ethnicity and neighborhood. Data on laxative use (specific brands, frequency of use, usual dose) were obtained by structured in-person interview.

Results Compared to women who never used a laxative, ever use of a phenolphthalein-containing laxative was not associated with an increased risk of invasive ovarian cancer (odds ratio (OR) $1.1,95 \%$ confidence interval $(\mathrm{CI}) 0.75,1.5$ ) or of borderline ovarian cancer (OR $0.75,95 \% \mathrm{CI} 0.37,1.5)$. Total days used, mean number of pills per day and cumulative dose were also unrelated to risk.

Conclusions This study provides some assurance that phenolphthalein-containing laxatives do not increase the risk of ovarian cancer in humans. These findings are of particular importance to those countries in which phenolphthalein is still used in over-the-counter medications. Published in 2003 John Wiley \& Sons, Ltd.
\end{abstract}

KEY WORDS - ovarian cancer; phenolphthalein; laxative

\section{INTRODUCTION}

Phenolphthalein was commonly used in many laxatives until the late 1990's when the United States Food and Drug Association reclassified phenolphthalein in over-the-counter medications as 'not generally recog-

\footnotetext{
* Correspondence to: G. S. Cooper, Epidemiology Branch A3-05, NIEHS, Durham, NC 27709, USA. E-mail: cooper1@ niehs.nih.gov ${ }^{\dagger}$ This article is a US Government work and is in the public domain in the USA.

Contract/grant sponsor: Division of Intramural Research, National Institute of Environmental Health Sciences.

Contract/grant number: CA 17054, CA 14089.

Contract/grant sponsor: National Institutes of Health.

Contract/grant number: CA 61132.
}

Published in 2003 by John Wiley \& Sons, Ltd. nized as safe'. ${ }^{1}$ This action was based on evidence from experimental studies in rats and mice in which treatment with phenolphthalein was shown to induce cancers at multiple sites, including the ovary. ${ }^{2,3}$ Although phenolphthalein-containing laxatives are no longer available in the United States or some European countries, ${ }^{4}$ they are still sold in other countries. ${ }^{5}$ We report data from a population-based casecontrol study of ovarian cancer conducted in Los Angeles, California, that included detailed information about type and duration of laxative use.

\section{MATERIALS AND METHODS}

Cases were identified by the Cancer Surveillance Program, the tumor registry covering all the residents of

Received 6 June 2002

Revised 19 November 2002

Accepted 3 January 2003 
Los Angeles County. Eligible cases were Englishspeaking non-Asian female residents of Los Angeles County with histologically confirmed invasive epithelial ovarian cancer or borderline (low malignant potential) ovarian cancer who were 18-74 years of age at diagnosis. Asian ovarian cancer patients were enrolled in a separate cross-cultural study that was being conducted by other members of the department. Cases and controls with previously diagnosed cancer (except non-melanoma skin cancer) were not eligible. The study protocol was approved by the Institutional Review Board of the University of Southern California, Keck School of Medicine. Details of subject recruitment were previously described. ${ }^{6}$

From October 1992 through October 1998, 1439 cases meeting the case definition were identified. Of these, 389 had died or were too ill to be interviewed, 74 could not be located, 62 could not be contacted because we did not receive permission from the patients' physicians, 36 had moved out of Los Angeles County and could not be interviewed in person and 189 declined to be interviewed. Interviews were conducted with 689 cases $(48 \%$ of the cases identified and $78 \%$ of the cases approached).

Controls were English-speaking non-Asian women with at least one intact ovary individually matched to cases on race/ethnicity (African-American, Latina, non-Latina white) and date of birth ( \pm 3 years). A neighborhood control was sought through a systematic canvas of the neighborhood. The first attempt to contact a potential control was made in person. Letters were left when no one was home, and follow-up by mail, telephone and further visits to the neighborhood continued until either an eligible control agreed to be interviewed or 450 housing units had been screened. For cases over the age of 65 , if no willing control could be found in the first 100 housing units, a control was simultaneously sought among a random sample of female residents of Los Angeles County over the age of 65 provided by the Health Care Financing Administration (HCFA). The HCFA control was matched to the case's race/ethnicity, date of birth (closest to that of the case) and zip code. By the end of the study, 645 controls were interviewed. The first eligible match was interviewed for $70 \%$ of the cases and the second match for another $21 \%$.

Approximately $20 \%$ of the older controls (those matched to cases over age 65) were identified from the HCFA registry and $80 \%$ were identified from the neighborhood canvas. The most notable difference between HCFA and neighborhood controls was that HCFA controls were somewhat older. When limited to controls ages 65 and over, $64 \%$ of the HCFA con-

Published in 2003 by John Wiley \& Sons, Ltd. trols were at least 70 years of age compared with $32 \%$ of the neighborhood controls. This difference would arise if older residents were less likely to respond to a neighborhood canvas. Cases and controls were interviewed in person using a comprehensive questionnaire covering medical, gynecological, reproductive and lifestyle histories. For both the case and her matching control, the questionnaire covered exposures and experiences that occurred up to the date corresponding to 2 months before the diagnosis date of the case. Calendars were used to aid in reconstructing reproductive and contraceptive histories using major life events to facilitate the recall of at least approximate dates.

The primary aim of the study was to examine galactose consumption and metabolism in relation to ovarian cancer risk. ${ }^{6}$ Our interest in phenolphthalein-containing laxatives was stimulated by the 1996 report from the National Toxicology Program ${ }^{2}$ and thus can be considered a secondary analysis. A section about laxative use was added to the interview when the study was underway and was completed by 356 $(52 \%)$ of the cases and $424(66 \%)$ of the controls. There were no notable demographic differences in the cases and controls who were interviewed before and after the section on laxatives was added.

Participants were asked if they had, before their reference date, 'ever taken a laxative to help you move your bowels' and if so, if they had ever taken more than six laxative pills in their lifetime. A list of 23 brands of laxatives was shown to the participants and the interviewer also probed for additional brands that were not on the list. Participants were asked to indicate the name of any laxative they had used, how old they were when they started using it, how many months they had used it, how often, on average, they used it (times per month) and how many tablets they usually took. The maximum number of different laxatives used by any subject was seven. We used the laxative-specific information to determine if each participant had ever used a phenolphthalein-containing laxative or had only used non-phenolphthalein-containing laxatives. For phenolphthalein-containing laxatives, we calculated total frequency of use (days), average number of pills per day, cumulative dose and age at first use. Cumulative dose was calculated as the summation across all phenolphthalein-containing laxatives of the product of $\mathrm{mg}$ phenolphthalein per pill times the average number of pills per day times number of days used.

We used the National Toxicology Program report as a source of information on specific phenolphthaleincontaining brands of laxatives ${ }^{3}$ and obtained dose

Pharmacoepidemiology and Drug Safety, 2004; 13: 35-39 
information from the Physicians' Desk Reference for Nonprescription Drugs. ${ }^{7}$ Use of at least one of the following phenolphthalein-containing laxatives was reported by study participants: Agoral, Alophen, Carter's Little Pills, Caroid, Colax, Correctol, Dialose, Doxidan, Espotabs, Evac-U-Gen, Evac-U-Lax, EvacU-Kwik, Ex-Lax, Feen-a-Mint, Kondrumel, LaxCaps, Lax-Pills, Medilax, Modane, Phenolax, Prulet and Unilax.

Although the study was designed as a matched case-control study, among the subjects interviewed after the section on laxatives was added, $30 \%$ of the cases $(n=107)$ did not have a matched control. This is higher than the corresponding figure for the full study (20\% of the cases without a matched control) and may reflect a decline in participation rates among controls in the later stages of the study. In order to retain these cases in the analysis, we used conditional logistic regression with 27 strata defined by nine age groups $(<=34,5$ year age groups from 35 to 69 and $>=70$ years) and three race/ethnicity groups (African-American, Latina and non-Latina white). Socioeconomic status was derived from mean income and education of neighborhood (4 groups). We continued to see a difference in individual educational level of our cases and controls after adjustment by our 4level socioeconomic status variable and so we also included the subject's actual educational level as a co-variate in the models to address this residual effect of education after adjustment. We adjusted for oral contraceptive use (ever, never), tubal ligation, parity $(0,1,2,3,4$ or more births), hysterectomy and infertility (ever had a problem getting pregnant). Since the risk estimates adjusted only for age, ethnicity and socioeconomic status were similar to those estimated by the more fully adjusted model, only the results from the latter are presented. There was no association between galactose consumption and metabolism and ovarian cancer risk in this study, ${ }^{6}$ so we did not control for these factors in this analysis. We also repeated the analyses excluding the cases without a matched control. The estimated associations were very similar, with slightly wider confidence intervals, to those of the full sample. The analyses for the full sample are presented.

Measures of frequency of use (total days, average number of pills per day and cumulative dose) and age use began were also examined. The referent group was 'no reported laxative use' in these models and women who had reported using only a non-phenolphthalein containing laxative were also included as a separate group. All analyses were run separately for invasive $(n=256)$ and low malignant potential

Published in 2003 by John Wiley \& Sons, Ltd. (borderline) $(n=100)$ ovarian cancer. Results are presented in terms of odds ratios (OR) as the measure of association and $95 \%$ confidence intervals $(\mathrm{CI})$.

\section{RESULTS}

The median age at diagnosis was 52 years (interquartile range 44-62 years) among ovarian cancer cases and 53 years (interquartile range $45-63$ years) among controls. The ethnic distribution of cases (10\% African-American, 17\% Latina and $72 \%$ nonLatina white) was similar to controls (7\% AfricanAmerican, 15\% Latina and 78\% non-Latina white).

A history of any laxative use (a total of seven or more laxative pills ever used before their reference date) was reported by $33 \%$ of the cases and $31 \%$ of the controls. Use of phenolphthalein-containing laxatives was essentially unrelated to the risk of invasive epithelial ovarian cancer (18\% ever use by cases, $15 \%$ by controls, adjusted OR $1.1,95 \%$ CI $0.75,1.5$ ) (Table 1). There was little evidence of an increased risk with total days used, mean number pills per day or cumulative dose. No association was observed between phenolphthalein exposure and risk of borderline ovarian cancer $(\mathrm{OR} 0.74,95 \% \mathrm{CI} 0.37,1.5$ for ever use of phenolphthalein-containing laxative).

\section{DISCUSSION}

We observed no association between the use of phenolphthalein-containing laxatives and ovarian cancer risk. We estimate, based on the prevalence among controls of the use of phenolphthalein-containing laxatives observed in this analysis (15\%), that the power for detecting a statistically significant association in this study was 0.40 for an OR of 1.5 and 0.87 for an OR of 2.0, assuming a two-sided alpha of 0.05.

Our results are similar to those from another population-based case-control study ${ }^{8}$ of epithelial ovarian cancer. Although there was some evidence of a dose-response effect in the other population-based study, the data from the present study do not show an increased risk with increasing exposure as measured by number of days used, average number of pills per day or cumulative dose. A recent hospital-based case-control study examined phenolphthalein-containing laxative use in relation to different types of cancer including leukemia and breast, lung, colon, endometrial and ovarian cancer. ${ }^{9}$ No associations were found with any of these cancers.

Chronic, high dose laxative use has been reported in cases of bulimia and other situations involving

Pharmacoepidemiology and Drug Safety, 2004; 13: 35-39 
Table 1. Phenolphthalein-containing laxative use and risk of invasive epithelial ovarian cancer*

\begin{tabular}{|c|c|c|c|c|c|c|c|}
\hline \multirow[b]{2}{*}{ Variable } & \multicolumn{2}{|c|}{ Cases } & \multicolumn{2}{|c|}{ Controls } & \multirow[b]{2}{*}{ OR } & \multirow[b]{2}{*}{$95 \% \mathrm{CI}$} & \multirow{2}{*}{$\begin{array}{l}\text { Overall } \\
p \text {-value }\end{array}$} \\
\hline & $n$ & $(\%)$ & $n$ & $(\%)$ & & & \\
\hline \multicolumn{8}{|l|}{ Laxative use $\ddagger$} \\
\hline None & 172 & (67) & 295 & (70) & 1.0 & referent & \multirow[t]{3}{*}{$(0.90)$} \\
\hline Non-phenolphthalein & 39 & (15) & 66 & (16) & 0.95 & $(0.66,1.4)$ & \\
\hline Phenolphthalein & 45 & (18) & 63 & (15) & 1.1 & $(0.75,1.5)$ & \\
\hline \multicolumn{8}{|c|}{ Phenolphthalein-containing laxatives (total days used) } \\
\hline $1-24$ & 13 & (29) & 23 & (37) & 0.98 & $(0.55,1.7)$ & \multirow[t]{3}{*}{$(0.84)$} \\
\hline $25-74$ & 16 & (36) & 23 & $(37)$ & 0.99 & $(0.57,1.7)$ & \\
\hline$\leq 75$ & 16 & (36) & 17 & $(27)$ & 1.2 & $(0.70,2.0)$ & \\
\hline \multicolumn{8}{|l|}{ Mean number pills per day } \\
\hline$\leq 1.0$ & 38 & (84) & 51 & $(81)$ & 1.1 & $(0.74,1.5)$ & \multirow[t]{2}{*}{$(0.80)$} \\
\hline$>1.0$ & 7 & (16) & 12 & (19) & 0.96 & $(0.42,2.2)$ & \\
\hline \multicolumn{8}{|l|}{ Cumulative dose $(\mathrm{mg}) \S$} \\
\hline$<3000$ & 20 & (44) & 27 & (43) & 1.1 & $(0.67,1.7)$ & \multirow[t]{3}{*}{$(0.99)$} \\
\hline $3000-10000$ & 13 & (29) & 19 & (30) & 1.0 & $(0.55,1.9)$ & \\
\hline$>10000$ & 12 & (27) & 17 & (27) & 1.0 & $(0.57,1.9)$ & \\
\hline \multicolumn{8}{|l|}{ Age first use (years) } \\
\hline$\leq 24$ & 19 & $(42)$ & 30 & $(48)$ & 1.0 & $(0.62,1.7)$ & \multirow[t]{3}{*}{$(0.28)$} \\
\hline$\overline{25}-34$ & 8 & (18) & 21 & (33) & 0.69 & $(0.33,1.5)$ & \\
\hline$\leq 35$ & 18 & $(40)$ & 12 & (19) & 1.4 & $(0.84,2.3)$ & \\
\hline
\end{tabular}

*Conditional logistic regression model within age-ethnic group strata, adjusting for socioeconomic status, education, oral contraceptive use, tubal ligation, number of live births and hysterectomy. No laxative use is the referent group, non-phenolphthalein containing laxative use is also included in the models. A total of 256 cases and 424 controls (low malignant potential cases excluded).

${ }^{\dagger}$ Overall test of association for the variable. $p$-value of the $-2 \log$ likelihood test comparing models with and without the variable.

${ }_{\ddagger}^{\ddagger}$ Use of 7 or more laxative pills before reference age.

${ }^{\S}$ Summation across all phenolphthalein-containing laxatives of product of $\mathrm{mg}$ phenolphthalein per pill times average number of pills per day times total number of days used.

laxative abuse. ${ }^{10,11}$ Only two women (both controls) in our study reported use of 10 or more pills per day, so we could not assess the effects of frequent (high dose) daily exposure.

The primary impetus for these epidemiologic studies was the experimental studies in rats and mice that provided evidence of the carcinogenic potential of phenolphthalein. ${ }^{2,3}$ In female mice, one of the affected sites was the ovary. The ovarian lesions (stromal cell hyperplasia and stromal cell tumor) were seen at the lowest dose of phenolphthalein used (3000 ppm in feed, administered continuously for 2 years). This dose is similar to the dose in humans consuming two or more phenolphthalein-containing laxative pills per day.

Although stromal cell tumors in the mouse are not directly related to human epithelial ovarian tumors, a mouse or rat model for epithelial ovarian cancer is not available. We focused on epithelial ovarian cancer because this form accounts for $85 \%$ of the ovarian cancer. ${ }^{16}$ Less than $10 \%$ of the ovarian cancer in humans is classified as sex-cord stromal tumors and to date, none of the epidemiologic studies have examined this form of cancer in relation to phenolphthalein exposure.

Published in 2003 by John Wiley \& Sons, Ltd.
The role of steroid and gonadotropin hormones in the etiology of ovarian cancer is not well understood. ${ }^{12,13}$ Mechanisms through which phenolphthalein could affect ovarian cancer include interaction with the estrogen-receptor ${ }^{14}$ and its potential influence on estrogen metabolism. In in vitro and in vivo studies in mice, Garner et al. ${ }^{15}$ recently reported that a metabolite of phenolphthalein, hydroxyphenolphthalein, inhibited $O$-methylation of catechol estrogens by catechol- $O$-methyltransferase.

Exposure misclassification could have occurred in our study because we relied on retrospectively collected information on laxative use. Because we did not observe an increased risk of ovarian cancer among users of laxatives that did not contain phenolphthalein, it is unlikely that our results were affected by confounding by indication for use or symptoms. A link between laxative use and ovarian cancer has not been widely discussed in the media, decreasing the likelihood that differential recall occurred in this study. Although interviewers were not blinded to case-control status, they were blinded to the phenolphthalein content of different laxatives. Thus it is unlikely that interviewer bias would have led to cases 


\section{KEY POINTS}

- Phenolphthalein, an active ingredient in some over-the-counter laxatives, was shown to be carcinogenic in experimental studies in rats and mice.

- Phenolphthalein is still used in some countries but has been voluntarily withdrawn from market in the US.

- In this population-based case-control study, we saw no evidence that use of phenolphthaleincontaining laxatives was associated with an increased risk of invasive or borderline epithelial ovarian cancer.

disproportionately reporting more phenolphthaleincontaining laxatives that were not on the pre-specified list. Selection bias could have occurred if phenolphthalein exposure was related to participation in the study, for example, by leading to a more aggressive disease and thus a greater likelihood of being too sick to participate. We cannot directly assess the potential for this bias, but we believe it is unlikely to account for our results.

This study, in combination with the two previous studies, ${ }^{8,9}$ provides some assurance that phenolphthalein-containing laxatives, as commonly used, do not increase the risk of epithelial ovarian cancer in humans. These findings are of particular importance to those countries in which phenolphthalein is still used in over-the-counter medications.

\section{ACKNOWLEDGMENTS}

We gratefully acknowledge the help of the ovarian cancer cases and controls whom we interviewed; without their help, no study would have been possible. The authors wish to acknowledge Mary Davis, Maureen Cairns, Cheryl Cooper, Jodi Senk, Elvira Tovar, Anna Gilmore, Audrey Cairns, Bernadette Hickman, Pattie Tang and Yan Kang Yu for their assistance with this study.

This study was funded by the Division of Intramural Research of the National Institute of Environmental Health Science and by grants CA 17054, CA 14089 and CA 61132 from the National Institutes of Health, and by a subcontract with the Public Health Institute. The subcontract is supported by the California Department of Health Services as part of its statewide cancer reporting program mandated by Health and Safety Code Section 103875 and 103885 . The ideas and opinions expressed herein are those of the authors, and no endorsement by the State of California, Department of Health Services or the Public Health Institute is intended or should be inferred. Additional support for cancer reporting was obtained from the National Cancer Institute at the National Institutes of Health under contract N01-PC-67010.

\section{REFERENCES}

1. Food and Drug Administration. Laxative drug products for over-the-counter human use. Fed Regist 1999; 64: 4535-4540.

2. Dunnick JK, Hailey JR. Phenolphthalein exposure causes multiple carcinogenic effects in experimental model systems. Cancer Res 1996; 56: 4922-4926.

3. National Toxicology Program. NTP technical report on the toxicology and carcinogenesis studies of phenolphthalein (CAS no. 77-09-8) in F344/N rats and B6C3F1R mice (feed studies). National Toxicology Program Technical Report Series. 1996; 465: 1-348. (NIN Publication Number 97-3990, available from the National Technical Information Service, Springfield, VA).

4. WHO Pharmaceuticals Newsletter. Laxatives containing phenolphthalein-voluntary withdrawal. 1998; 1-2: 4-15.

5. De Smet PH, Wagenaar HW. Weight reduction preparations from Thailand: unexpected complication for the traveler. Ned Tijdschr Geneeskd 1988; 142: 2798-2800.

6. Cozen W, Peters RK, Reichardt JKV, et al. Galactose-1-phosphate uridyl transferase (GALT) genotype and phenotype, galactose consumption and the risk of borderline and invasive ovarian cancer. Cancer Causes Control 2002; 13: 113-120.

7. Physicians' Desk Reference for Nonprescription Drugs and Dietary Supplements. 11th edition. Medical Economics Company, Inc: Montvale, NJ, 1990.

8. Cooper GS, Longnecker MP, Sandler DP, Ness RB. Risk of ovarian cancer in relation to use of phenolphthalein-containing laxatives. Br J Cancer 2000; 83: 404-406.

9. Coogan PF, Rosenberg L, Palmer JR, et al. Phenolphthalein laxatives and risk of cancer. J Natl Cancer Inst 2000; 92: 1943-1944.

10. Bo-Linn GW, Santa Ana CA, Morawski SG, Fordtran JS. Purging and caloric absorption in bulimic patients and normal women. Ann Intern Med 1983; 99: 14-17.

11. Bytzer P, Stokholm M, Andersen I, Klitgaard NA, Schaffalitzky de Muckadell OB. Prevalence of surreptitious laxative abuse in patients with diarrhoea of uncertain origin: a cost benefit analysis of a screening procedure. Gut 1989; 30: 13791384.

12. Cramer DW, Hutchinson GB, Welch WR. Determinants of ovarian cancer risk. II. Inferences regarding pathogenesis. J Natl Cancer Inst 1983; 71: 717-721.

13. Risch HA. Hormonal etiology of epithelial ovarian cancer, with a hypothesis concerning the role of androgens and progesterone. J Natl Cancer Inst 1998; 90: 1774-1786.

14. Ravdin PM, van Beurden M, Jordan VC. Estrogenic effects of phenolphthalein on human breast cancer cells in vitro. Breast Cancer Res Treat 1987; 9: 151-154.

15. Garner CE, Matthews HB, Burka LT. Phenolphthalein metabolite inhibits catechol-o-methyltranseferase-mediated metabolism of catechol estogens: a possible mechanism for carcinogenicity. Toxic Applied Pharmacol 2000; 162: 124-131.

16. Scully RE. Classification of human ovarian tumors. Environ Health Perspect 1987; 73: 15-24. 